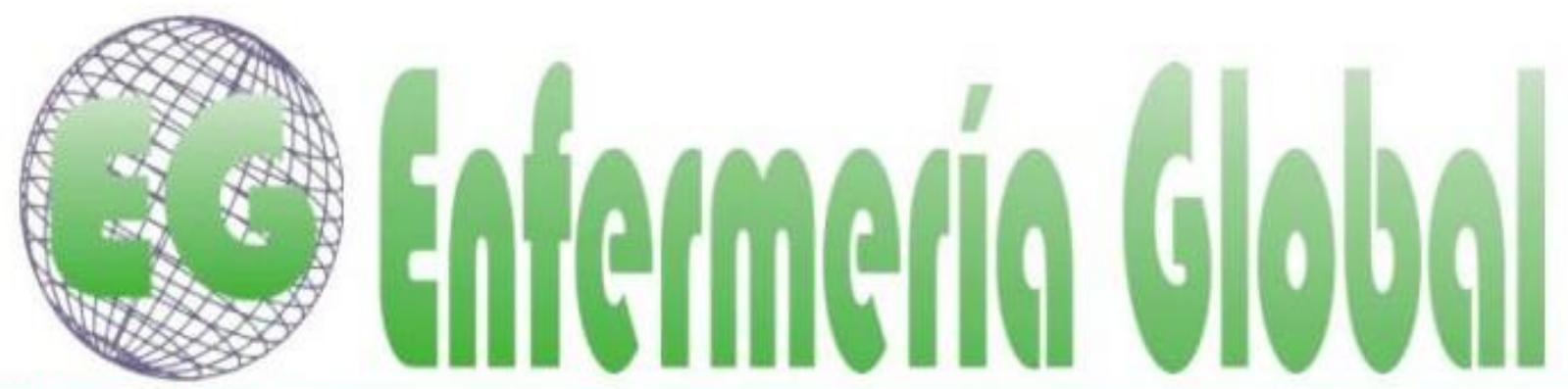

\title{
Estudio en familias: factores ambientales y culturales asociados al sobrepeso y obesidad
}

Study of families: Environmental and Cultural Factors associated with Overweight and Obesity

\section{*Gutiérrez Valverde, Juana Mercedes *Guevara Valtier, Milton Carlos *Enríquez Reyna, María Cristina *Paz Morales, María de los Ángeles *Hernández del Ángel, Martha Arely **Landeros Olvera, Erick Alberto}

*Universidad Autónoma de Nuevo León, Facultad de Enfermería. Monterrey, N.L. E-mail: milton.guevarav@uanl.mx ***Benemérita Universidad Autónoma de Puebla, Facultad de Enfermería. México.

http://dx.doi.org/10.6018/eglobal.16.1.253811

\section{RESUMEN}

Objetivo: Determinar si el sobrepeso y la obesidad en los integrantes de la familia están relacionados con los factores ambientales y culturales relativos al funcionamiento de la familia.

Método: Estudio descriptivo correlacional. La muestra fue constituida por 198 integrantes de familias. Se aplicaron una Cédula de Datos Generales, una Escala para Evaluar las Relaciones Intrafamiliares, el Cuestionario de Elección de Alimentos y el Cuestionario de Actividad Física.

Resultados: El 8.6\% de los participantes mayores de 18 años presentó sobrepeso y el $46.5 \%$ algún grado de obesidad. El índice de masa corporal de los adultos se relacionó con las kilocalorías totales producto de la actividad física $(\mathrm{rs}=.594 ; \mathrm{p}<.01)$ y con las subescalas ecología de la alimentación $(\mathrm{rs}=.160 ; \mathrm{p}<.05)$ y valor nutritivo $(\mathrm{r} s=.242 ; \mathrm{p}<.01)$. Los factores culturales no se asociaron con el índice de masa corporal $(r s=.242 ; p>.01)$.

Conclusiones: En esta muestra el Sobrepeso/Obesidad se asoció con los factores ambientales.

Palabras clave: Familia; Cultura; Ambiente; Sobrepeso; Obesidad

\section{ABSTRACT}

Objective: Determine whether overweight and obesity in family members are related to environmental and cultural factors regarding the functioning of families. 
Method: Descriptive Correlational Study. The sample consisted of 198 family members. A General Data Card, a Scale to Evaluate Intra-family Relations, the Food Selection Questionnaire, and the Physical Activity Questionnaire were used.

Results: $8.6 \%$ of the participants 18 years old or older were overweight, and $46.5 \%$ had some degree of obesity. The Body Mass Index of adults was related to total kilocalories as a product of physical activity $(\mathrm{r} s=.594 ; \mathrm{p}<.01)$ and with the subscales, eating ecology $(\mathrm{r} s=.160 ; \mathrm{p}<.05)$ and nutritional value $(r s=.242 ; p<.01)$. Cultural values were not associated with the Body Mass Index ( $r s=.242 ; p>.01)$.

Conclusions: In this sample, Overweight/Obesity was associated with environmental factors.

Keywords: Family; Culture; Environment; Overweight; Obesity.

\section{INTRODUCCIÓN}

El sobrepeso y obesidad (SO) son enfermedades crónicas multifactoriales en la que están implicados factores genéticos y ambientales, manifestadas por expansión patológica de los depósitos adiposos corporales ${ }^{(1)}$ y con una acumulación anormal o excesiva de grasa, caracterizada por un índice de masa corporal (IMC) superior a 25 $\mathrm{kg} / \mathrm{m}^{2}$ (sobrepeso) $0 \geq 30 \mathrm{~kg} / \mathrm{m}^{2}$ (obesidad) en personas adultas. ${ }^{(2)}$

El SO es un problema de salud pública por su impacto sobre la morbi-mortalidad, calidad de vida y gasto sanitario. ${ }^{(3)} \mathrm{A}$ nivel mundial la obesidad ocupa el quinto lugar de mortalidad y cada año fallecen 2.8 millones de adultos por esta causa. La carga de morbilidad atribuible al SO puede distinguirse de acuerdo a su etiología en un $44 \%$ por diabetes, $23 \%$ por cardiopatías isquémicas y entre el $7 \%$ y el $41 \%$, por algunos cánceres. ${ }^{(2)}$ En diversos países la prevalencia de SO ha tenido un aumento notable, debido a cambios sociales como una mayor disponibilidad de alimentos y un progresivo descenso de la actividad física. ${ }^{(4)}$

El caso específico de Nuevo León, México, es preocupante por presentar valores similares o superiores a la media nacional. En Nuevo León, el SO afecta a 39.3\% de los adolescentes entre 12 y 19 años; y al $71.7 \%$ de la población adulta de 20 a 59 años. Así mismo, la prevalencia de obesidad abdominal en esta misma población (2059 años) es de $66.4 \%$ para los hombres y el $76.2 \%$ para las mujeres, siendo las últimas las que presentan una mayor prevalencia con una diferencia de $9.8 \%$ en relación a los hombres. ${ }^{(5)}$ Pese a que la presencia generalizada de SO en una población puede tener un origen multifactorial; pudiera estar enmarcada y diseminarse por un estilo de vida familiar inapropiado.

La familia se define como un grupo social que aglutina a sus integrantes en función del parentesco ya sea consanguíneo, por matrimonio o adopción; los individuos viven juntos por un período indefinido de tiempo en el que asumen responsabilidades que influyen en la preservación de la vida humana, desarrollo, salud, bienestar. ${ }^{(6)}$ En las familias, los estilos de vida se aprenden y fomentan entre sus miembros a lo largo de la vida. Los niños y adolescentes son más susceptibles a aprender estilos de vida saludables o no saludables que se presentan en el medio que se desarrollan. En la edad adulta, esto contribuye al desarrollo de enfermedades crónico degenerativas como el SO. ${ }^{(7)}$

A nivel social, otra influencia que podría llevar a desarrollar SO es la cultura. La cultura de alimentación influye a nivel individual a través del funcionamiento familiar. Se refiere a la forma en que los individuos se comportan entre sí, es decir, un conjunto de vínculos familiares que dispone de características y comportamientos 
identificados que pueden ser valorados. ${ }^{(8)}$ En la literatura existe una variedad de instrumentos y modelos que pueden ser usados para la valoración de esos vínculos, características y comportamientos asociados a la familia. Un ejemplo de estos es el Modelo Calgary de Valoración Familiar. ${ }^{(9)}$

De acuerdo a los datos existentes de SO y los problemas de salud consecuentes, es importante conocer las características y actividades de los integrantes de la familia que pudieran influir en el desarrollo del SO. Este trabajo tuvo como propósito determinar si el $\mathrm{SO}$ en los integrantes de la familia está relacionado con los factores ambientales y culturales relativos al funcionamiento de la familia.

\section{METODOLOGIA}

El diseño fue descriptivo correlacional, transversal. ${ }^{(10)}$ La población de interés la constituyeron personas con y sin SO y familiares que viven bajo el mismo techo. Se entrevistó un total de 70 familias, con 198 integrantes. La muestra fue calculada por medio del paquete n'Query Advisor Versión 4.

El estudio cumplió con las disposiciones del Comité de Ética e Investigación de la institución auspiciadora.

\section{Pruebas de lápiz y papel}

\section{Factores Ambientales}

Los factores ambientales (actividad física y alimentación) se midieron mediante el Cuestionario de Actividad Física (CAF), validado en población mexicana con valores aceptables de reproducibilidad (Correlación intraclase $=0.62, p<0.001$ ) y sensibilidad $(p<0.01)$. El cuestionario se elaboró para ser un auto reporte dividido de acuerdo a las horas del día en periodos de 15 minutos con el fin de indicar la actividad(es) realizada(s) por la persona. Las actividades realizadas por los integrantes de la familia podrían variar desde actividades muy ligeras como dormir, hasta actividades 0 deportes muy intensos como aerobics de impacto moderado o practicar fútbol. En el cuestionario se realiza un registro de tres días de la actividad física de una persona divididos en periodos de 15 minutos que posteriormente se transforman a kilocalorías gastadas (Kcal) con la formula Kcal = valor de la actividad en MET $\mathrm{x}$ peso en $\mathrm{kg} \times$ tiempo en horas (CAF). ${ }^{(11)}$

La alimentación fue medida a través del Cuestionario de Elección de Alimentos $(\mathrm{CEA})^{(12)}$ en su versión en español, tiene un total de 36 preguntas, divididas en 9 dimensiones (salud, estado de ánimo, comodidad, atracción sensorial, contenido natural, precio, peso, familiaridad y asuntos éticos), cada pregunta tiene opciones de respuesta que van de 1 o muy importante, hasta 4 muy importante, este cuestionario ha mostrado una consistencia interna aceptable con valores alpha de cronbach de 0.72 a $0.86 .^{(13)}$

\section{Factores culturales}

El factor cultural (costumbres) fue medido a través de la Escala de Evaluación de las Relaciones Intrafamiliares (EERI), instrumento integrado por 56 reactivos. Con una confiabilidad de .93 e integrada por tres dimensiones: unión y apoyo, expresión y dificultades. Se trata de una escala autoaplicable con cinco opciones de respuesta 
que varían de totalmente de Acuerdo $(T A=5)$ a totalmente en Desacuerdo $(T D=1) .{ }^{(14)}$ Los factores culturales religión y nivel educativo fueron registrados en una cédula de datos personales (CDP) que también incluyó los datos sociodemográficos como edad, sexo, estado civil, religión y años de educación formal.

\section{Mediciones}

Se midió la talla con estadímetro SECA 213 y el peso con báscula SECA 700. A partir del peso y la talla se estimó el IMC. Para clasificar a los adultos con SO se calculó el IMC según las recomendaciones de la Organización Mundial de la Salud. Los niños se clasificaron con bajo peso cuando su percentil era menor del $14 \%$, con peso normal cuando su resultado fue de 14.1 a $16.5 \%$ de 16.6 a $18.2 \%$ se categorizaron con sobrepeso y obesidad cuando los valores eran superiores a $18.2 \%$.

\section{Procedimientos de recolección de datos}

Una vez obtenidos los permisos en cada institución, se contactó a personas en un centro de salud en la sala de espera que cumplieran con los criterios de inclusión. Se explicó a los candidatos la identidad y procedencia educativa del investigador, los objetivos del estudio, el tipo de preguntas y mediciones. Se hacia énfasis sobre la importancia de contactar por lo menos a dos de los integrantes de su familia que vivieran en la misma casa, se les ofreció la opción de realizar visita domiciliaria o cita para nuevo contacto en el centro de salud. De acuerdo a la alternativa tomada por las personas para ser parte del estudio y bajo autorización en un consentimiento informado, se realizaron las mediciones, el llenado de los cuestionarios y finalmente se agradeció a cada participante por su participación en el estudio.

\section{Análisis de datos}

Los datos se analizaron con el software SPSS versión 21.0. Se utilizaron frecuencias y porcentajes para la descripción de la muestra. Se evaluó la distribución de los datos y se corrieron análisis de correlación. El valor de alfa fue de .05.

\section{RESULTADOS}

\section{Características generales de la muestra}

Las familias estudiadas estuvieron compuestas por padre, madre, hijo(a), sobrino(a), hermano(a), abuelo(a), tío(a), suegro(a), nieto(a) que consultaron en un centro de salud y que cohabitaban un solo domicilio. Se analizaron 198 integrantes de 70 familias.

Las características generales de los integrantes de la familia (edad, sexo, estado civil, religión y años de escolaridad), se presentan con medidas de tendencia central y de dispersión. La mayoría de los participantes fueron adultos de entre 18 y 85 años $(93.3 \%)$ quienes eran responsables o padres de familia de menores de edad de entre 11 y 17 años (6.7\%). La edad promedio de los participantes fue de 35.22 años $(D E=13.3$, Rango=11-85). El $63.8 \%$ de la muestra fueron mujeres. El promedio de escolaridad fue de 12 años. El $51.4 \%$ de los participantes refirió ser de estado civil casado y el $89 \%$ profesa la religión católica. 
Con respecto a la prevalencia de SO, el $8.6 \%$ de los participantes mayores de 18 años presentó sobrepeso y el $46.5 \%$ algún grado de obesidad (Tabla I).

Tabla I. Clasificación del IMC de adultos de acuerdo a los parámetros de la OMS (2012).

\begin{tabular}{lccc}
\hline Categoría & IMC $(\mathrm{kg} / \mathrm{m} 2)$ & $\mathrm{f}$ & $\%$ \\
\hline Normal & $18.5-24.9$ & 30 & 15.1 \\
Sobrepeso & $25-25.8$ & 17 & 8.6 \\
Obesidad & $25.9-29.9$ & 92 & 46.5 \\
Obesidad I & $30.0-34.9$ & 56 & 28.3 \\
Obesidad II & $35.0-39.9$ & 3 & 1.5 \\
\hline Total & & 198 & 100 \\
\hline
\end{tabular}

Nota: IMC=Índice de masa corporal; $\mathrm{f}=$ frecuencia; $\%=$ porcentaje $\mathbf{n}=198$

Con respecto al rol familiar de los participantes, el $3.5 \%$ (7) de los hijos de presentaron sobrepeso, el $9.5 \%$ (19) de los padres de familia y el $14.6 \%$ (29) de las madres de familia presentaron obesidad, como se muestra en la tabla II.

Tabla II. Frecuencias y porcentajes del IMC de los integrantes de la familia por rol dentro de la familia.

\begin{tabular}{|c|c|c|c|c|c|c|c|c|c|c|c|}
\hline \multirow{2}{*}{$\begin{array}{l}\text { Integrante } \\
\text { de la familia }\end{array}$} & \multicolumn{2}{|c|}{$\mathbf{N}$} & \multicolumn{2}{|c|}{ SP } & \multicolumn{2}{|c|}{0} & \multicolumn{2}{|c|}{ Ol } & \multicolumn{2}{|c|}{ OlI } & \multirow[t]{2}{*}{ TOTAL } \\
\hline & $f$ & $\%$ & $f$ & $\%$ & $f$ & $\%$ & $f$ & $\%$ & $f$ & $\%$ & \\
\hline Padre & 3 & 1.5 & 2 & 1.0 & 19 & 9.5 & 11 & 5.5 & 3 & 1.5 & 38 \\
\hline Madre & 8 & 4.0 & 5 & 2.5 & 29 & 14.6 & 14 & 7.0 & - & - & 56 \\
\hline Hijo (a) & 7 & 3.5 & 7 & 3.3 & 24 & 12.1 & 14 & 7.5 & - & - & 52 \\
\hline Hermano (a) & 5 & 2.5 & - & - & 7 & 3.5 & 6 & 3.0 & - & - & 18 \\
\hline Abuelo (a) & 1 & 0.5 & - & - & - & - & 1 & 0.5 & - & - & 2 \\
\hline Tío (a) & 0 & 0 & 1 & 0.5 & 2 & 1.0 & 1 & 0.5 & - & - & 4 \\
\hline Sobrino (a) & 3 & 1.5 & - & - & 3 & 1.5 & 2 & 1.0 & - & - & 8 \\
\hline Suegro (a) & 1 & 0.5 & - & - & 2 & 1.0 & 4 & 2.0 & - & - & 7 \\
\hline Nuera & 1 & 1 & 1 & 0.5 & 3 & 1.5 & 0 & 0 & - & - & 5 \\
\hline Cuñado (a) & 1 & 1 & 0 & 0 & 2 & 1.0 & 1 & 0.5 & - & - & 4 \\
\hline Nieto & 0 & 0 & 1 & 0.5 & 1 & 0 & 2 & 1.0 & - & - & 4 \\
\hline Total & 30 & 16 & 17 & 8.3 & 92 & 45.7 & 56 & 28.5 & 3 & 1.5 & 198 \\
\hline
\end{tabular}

$\mathbf{N}=$ Normal, $\mathbf{S P}=$ Sobrepeso, O=Obesidad, Ol=Obesidad I, OII=Obesidad II. $\mathbf{n = 1 9 8}$

En relación a los menores de edad, $66.6 \%$ (8) presentó obesidad y $16.7 \%$ (2) sobrepeso y peso normal, respectivamente. 


\section{Análisis inferencial}

Previo al análisis inferencial se realizó la prueba Kolmogorov-Smirnov con corrección de Lilliefors para determinar la distribución de los datos. Debido a la falta de normalidad ( $p \leq .001)$, se decidió el uso de estadística no paramétrica.

Con respecto a la actividad física en periodos de tres horas, el $33.8 \%$ (71) refirió "permanecer sentado", el 29.5\% (62) realiza actividad física moderada (caminar, ir de compras, conducir moto, etc.) y el $18.6 \%$ (39) trabajo manual ligero (aseo/limpieza de casa).

Con el fin de conocer la asociación del IMC de los participantes con los factores ambientales (actividad física y alimentación), se utilizó prueba de correlación Spearman para el conjunto de variables. Se encontró que la subescala 7 se correlacionó positiva y significativamente con el IMC de los adultos $\left(r_{\mathrm{s}}=.142 ; p=.047\right)$, las Kcal totales con el IMC de los adultos $\left(r_{s}=.549 ; p=.000\right)$, se. La subescala 8 con el IMC de los adultos $\left(r_{\mathrm{s}}=-.174 ; p=.014\right)$ y la subescala 5 con el IMC de los menores $\left(r_{s}=\right.$ $-.591 ; p=.043)$ se correlacionaron de forma negativa y significativa. No se encontró asociación entre el IMC con los factores culturales ( $p>.05)$.

\section{DISCUSIÓN}

En el presente estudio, más del $80 \%$ de los integrantes de la familia fueron mayores de 18 años, los cuales presentaron IMC elevados, y además por lo menos un integrante de la familia tenía SO, esto concuerda con el reporte de Rossi de los Ángeles, Castellanos Reyes y Saint-Hilaire Zayas ${ }^{(15)}$ quienes mencionan que algunos integrantes de la familia que viven bajo un mismo techo se interrelacionan entre sí influyendo en sus integrantes el fomento de estilos de vida poco saludables como el consumo excesivo de kilocalorías y poca o nula actividad física, lo que contribuye al aumento de peso. El sexo femenino fue el predominante en todo el estudio, con el rol de madre de familia, siendo ese rol en el que recae la administración de los recursos del hogar y las decisiones alimenticias. Es en el sexo femenino donde recaen la mayoría de las obligaciones del hogar y ejercen un rol protagónico para el logro de cambios en los estilos de vida, especialmente de los menores de edad. ${ }^{(16)}$

Con respecto a la prevalencia de SO se encontró que más del $40 \%$ de los adultos padecían obesidad, el $10 \%$ sobrepeso, y más del $60 \%$ de los menores de edad tenían obesidad, lo cual es considerado alto. En contraste, en población infantil de España se han encontrado prevalencias desde un $12.4 \%$ en el estudio EnKid, $15 \%$ de sobrepeso y $10.5 \%$ de obesidad, en el estado de Córdoba, y en el estudio Aladino cifras de hasta un $26.1 \%{ }^{(17,18)}$ Esta situación puede explicarse a partir de los determinantes sociales de la salud dado que los resultados encontrados en ambos estudios son muestras de lugares contextuales, étnicos, culturales y con costumbres que en el interior de la familia pueden ser distintos. Es posible que las prácticas de selección, preparación/consumo de alimentos sean distintas, sin embargo, a pesar de las marcadas diferencias de obesidad infantil encontradas en ambos estudios se reconoce una situación de importancia de peligro para la salud por lo que un argumento que puede explicar este fenómeno parte de la constante exposición a la publicidad de alimentos y bebidas de alto contenido calórico, ricos en grasas, azúcar y sal, así como una gran oferta de este tipo de alimentos en todo lugar al que los niños concurren, ha inducido un cambio importante en el patrón de alimentación infantil. ${ }^{(19)}$ 
Aunado a lo anterior, las diferencias encontradas con respecto a las cifras de sobrepeso y obesidad encontradas puede deberse a los criterios metodológicos que se utilizarón para el diagnóstico de sobrepeso u obesidad, es decir; mientras que algunos autores utilizan como punto de cohorte para el diagnóstico criterios basados en percentiles (percentil 90 y 97), otros hacen uso de los criterios de la American Task Force, de la Organización Mundial de la Salud o cifras referidas por los padres de Familia. ${ }^{(18)}$

Con respecto al propósito del estudio en el que se planeó conocer si el SO en los integrantes de la familia está relacionado con los factores ambientales y culturales relativos al funcionamiento de la familia, se encontró que los factores ambientales actividad física y alimentación tienen un papel importante en este sentido, es decir guardan relación. Esta situación puede explicarse a partir de las representaciones sociales creadas por los integrantes de la familia a diversas actividades de importancia como la alimentación y la práctica de actividad física. En el caso de la alimentación esta tiene una representación social de importancia debido a que permite la sobrevivencia, es una fuente necesaria de energía que se refleja directamente en la salud, el acto de comer o alimentarse es visto desde la perspectiva de las familias como un indicador de bienestar y equilibrio. ${ }^{(20)}$

A pesar de lo anterior llama la atención que dicha relación fue dada posiblemente por las cifras elevadas de obesidad, un posible consumo de calorías superior a las necesidades requeridas y un nivel inferior de actividad física por los integrantes de la familia, lo que indique tal vez que su percepción con respecto al estatus de salud familiar y riesgo para el desarrollo de complicaciones futuras sea subvalorado, estos últimos datos son apoyados con los resultados de Cabello y Zúñiga ${ }^{(20)}$ quienes encontraron que las personas con obesidad manifiestan sensaciones de estar felices o contentos con el hecho de tener cuerpos grandes o con exceso de grasa corporal.

Otros argumentos que pueden explicar los hallazgos identificados sugieren que la génesis de la obesidad se asocia con eventos de vida estresantes y con la utilización de la comida como refugio para compensar frustraciones, tristeza, necesidades, temores que puedan surgir en el interior de las familias como parte del resultado de las interacciones al interior y exterior de las mismas representadas por el medio ambiente que rodea la alimentación en la familia en el que se observan los estilos de vida, apoyo social, comportamientos del comer, sentimientos, actitudes, disponibilidad de alimentos y sitios para prácticas actividad física; así como aspectos culturales como la religión y las costumbres. ${ }^{(19,21)}$

Con base a la variable costumbres, no se identificó relación lo cual podría deberse a aspectos metodológicos como el tipo de cuestionario utilizado o al diseño del estudio, esto último debido a que a partir de teoría fenomenológica o etnográfica sea posible tener un acercamiento estrecho con las familias y comprender a profundidad la relación mencionada en el propósito de este estudio. Cabe señalar que la génesis de la obesidad tiene una fuerte explicación cultural, es decir, desde la perspectiva de Albert Bandura, los niños aprenden e imitan comportamientos de iguales, u otros miembros mayores de la familia, como hermanos, madre, padre, tíos o abuelos que pueden ser caracterizados en costumbres como el hecho de alimentarse de forma insana o no realizar actividad física de importancia para la salud, otros aspectos culturales que son de importancia para la configuración del aprendizaje transformado en conductas familiares son el estatus socioeconómico, lugar de vivienda, desayuno o 
cena, ausencia de control estricto sobre la elección de los alimentos y una escasa presión familiar para el consumo de alimentos. ${ }^{(22)}$

Los resultados de este estudio cobran interés para la formulación de propuestas de investigación que intenten efectuar cambios en los indicadores de salud de las familias, e inclusive los datos podrían ser utilizados para ejercer influencia en la toma de decisiones en el primer nivel de atención para la salud, como evidencia de guías de salud e ideas para el diseño de investigaciones futuras.

\section{CONCLUSIONES}

Es de suma importancia la valoración de la función de la familia como una institución en la que se aprenden, socializan conductas, en este caso las de tipo alimentarias y de actividad física, el caso específico de los padres de familia juega un papel de suma importancia ya que son los principales modelos a seguir por parte de los hijos o menores de edad en el interior de la familia, por tanto son responsables de enseñar estilos de vida saludables para la prevención de enfermedades relacionadas con el Sobrepeso y la Obesidad (SO)

De acuerdo con los resultados encontrados, en esta muestra de 70 familias, el SO se relacionó con factores ambientales como la actividad física, la ecología de la alimentación, el control de peso y el valor nutritivo de los alimentos. En contraste, factores culturales como las costumbres al respecto de la unión, apoyo, expresión y dificultades no se asociaron con la presencia de SO.

Se encontraron porcentajes altos de sobrepeso/obesidad y prácticas de actividad física de poco beneficio para la salud, lo anterior tanto en adultos como en menores de edad que formaban las familias estudiadas.

\section{REFERENCIAS}

(1) López-Villalta Lozano MJ, Soto González A. Actualización en Obesidad, Complejo Hospitalario Universitario de A. Coruña. 2010;17:101-10. Disponible en: http://www.agamfec.com/wp/wp-

content/uploads/2014/08/vol17 n2 Para saber de 01.pdf

(2) Organización Mundial de la Salud [OMS]. Ginebra, Suiza: OMS. Sobrepeso y Obesidad: Conceptos. Disponible en: http://www.who.int/mediacentre/factsheets/fs311/es/

(3) James WP. The epidemiology of obesity: the size of the problem. J Intern Med. 2008;263(4):336-52. Disponible en: http://onlinelibrary.wiley.com/doi/10.1111/j.13652796.2008.01922.x/epdf

(4) Santos JL, Martínez JA, Pérez F, Albala C. Epidemiologia genética de la obesidad: estudios familiares. Revista Médica de Chile, 2005;133(3):349:361. Disponible en: http://www.scielo.cl/pdf/rmc/v133n3/art12.pdf

(5) Instituto Nacional de Salud Pública - ENSANUT [Internet]. Cuernavaca, México. Resultados por entidad federativa: Nuevo León. Disponible en: http://ensanut.insp.mx/informes/NuevoLeon-OCT.pdf

(6) Instituto Interamericano del Niño, la Niña y Adolescentes [Internet]. Uruguay: IIN. Disponible en: www.iin.oea.org/Cursos a distancia/Lectura\%2012 UT 1.pdf

(7) Biblioteca del Congreso Nacional de Chile [Internet]. Chile. La familia: concepto Guía de Educación Cívica. Disponible en: http://www.bcn.cl/ecivica/concefamil/ 
(8) Tschann JM, Gregorich SE, Penilla C, Pasch LA, Groat C, Flores E, et al. Parental feeding practices in Mexican American families: initial test of an expanded measure. Int $J$ Behav Nutr Phys Act. 2013;10:6. Disponible en: http://www.ncbi.nlm.nih.gov/pmc/articles/PMC3552721/pdf/1479-5868-10-6.pdf

(9) Wright LM, Leahey M. Nurses and Families: A Guide to Family Assessment and Intervention. 5ta Edition. Philadelphia: F. A. Davis Company; 2009.

(10) Burns N, Grove SK. Investigación en Enfermería. 5ta Edición. España: Elsevier; 2004.

(11) López-Alvarenga JC, Reyes-Díaz S, Castillo-Martínez L, Dávalos-lbáñez A, González-Barranco J. Reproducibilidad y sensibilidad de un cuestionario de actividad física en población mexicana. Salud Pública de México. 2001;43(4):306-312.

(12) Steptoe A, Pollard TM, Wardle J. Development of a measure of the motives underlying the selection of food: the food choice questionnaire. Appetite. 1995;25(3):264-84.

Disponible

en:

https://www.ucl.ac.uk/hbrc/resources/resources eb/Steptoe 1995 FCQ Appetite.pdf

(13) Jáuregui-Lobera I, Bolaños Ríos P. What motivates the consumer's food choice? Nutr Hosp. 2011;26(6):1313-1321.

(14) Rivera-Heredia ME, Andrade-Palos P. Escala de evaluación de las relaciones intrafamiliares (E. R. I.). Uaricha Revista de Psicología, 2010;14:12-29. Disponible en: http://www.revistauaricha.umich.mx/Articulos/Uaricha 14 012-029.pdf

(15) Rossi de los Ángeles YM, Castellanos Reyes ND, Saint-Hilaire Zayas EY. Prevalencia de sobrepeso y obesidad en las familias de la zona sur de Santiago determinado por índice de masa corporal e índice nutricional. Revista Médica Dominicana. 2011;72(2):139-143. Disponible en: https://www.bvs.org.do/revistas/rmd/2011/72/02/RMD-2011-72-02-139-143.pdf

(16) González Jiménez E, Aguilar Cordero MJ, García García CJ, García López P, Álvarez Ferre J, Padilla López A, et al. Influencia del entorno familiar en el desarrollo del sobrepeso y la obesidad en una población de escolares de Granada (España). Nutr Hosp. 2012;27(1):177-184.

(17) Alba-Martín R. Prevalencia de obesidad infantil y hábitos alimentarios en educación primaria. Enfermería Global. 2016;(42):40-51. Disponible en: http://revistas.um.es/eglobal/article/viewFile/212531/191191

(18) Martínez-Álvarez JR, Villarino Marín RM, Calle Purón ME, Marrodán Serrano MD. Obesidad infantil en España: hasta qué punto es un problema de salud pública o sobre la fiabilidad de las encuestas. Nutr. clin. diet. hosp. 2013; 33(2):80-88. Disponible

en:

http://www.fundacionsaborysalud.com/web/portal/images/documentacion/OBESIDADINFANTIL.pdf

(19) Domínguez-Vásquez $P$, Olivares S, Santos JL. Influencia familiar sobre la conducta alimentaria y su relación con lo obesidad infantil. Archivos Latinoamericanos de Nutrición. 2008;58(3):349. Disponible http://repositorio.uchile.cl/bitstream/handle/2250/123925/Dominguez p.pdf?sequence $=1$

(20) Cabello Garza ML, Zuñiga Zárate JG. Aspectos intrapersonales y familiares asociados a la obesidad: un análisis fenomenológico. CIENCIA UANL. 2007;X(2):183188. Disponible en: eprints.uanl.mx/1770/1/OBESIDAD.pdf

(21) Kottak, CP. Antropología Cultural. 14ª Ed. Madrid: McGraw-Hill; 2014.

(22) Cislak A, Safron M, Pratt M, Gaspar T, Luszczynska A. Family-related predictors of body weight and weight-related behaviours among children and adolescents: a systematic umbrella review.Child Care Health Dev. 2012;38(3):321-31. Disponible en: http://www.bago.com/BagoArg/Biblio/pediatweb635.htm 
Recibido: 16 de marzo 2016;

Aceptado: 28 de mayo 2016 